\title{
Enunciación
}

http://revistas.udistrital.edu.co/ojs/index.php/enunc

DOI: http://dx.doi.org/10.14483/udistrital.jour.enunc.2014.2.a13

\section{Los retos actuales de la alfabetización académica: estado de la cuestión y últimas investigaciones*}

\author{
Montserrat Castelló ${ }^{1}$
}

Para citar este artículo: Castelló, M. (2014). Los retos actuales de la alfabetización académica: estado de la cuestión y últimas investigaciones. Enunciación, 19(2), 346-365.

\section{Resumen}

En este capítulo nos proponemos definir los retos actuales del estudio de la alfabetización académica analizando su carácter situado, social e interdisciplinar y su impacto en las situaciones educativas que se promueven en la universidad. Esta caracterización no ha empezado a ser ampliamente aceptada por la comunidad científica hasta los últimos veinte años y es, por tanto, heredera de tradiciones teóricas y epistemológicas anteriores. En primer lugar haremos un repaso al estado actual de la investigación en escritura académica situando geográfica, conceptual y epistemológicamente los enfoques predominantes. En un segundo momento analizaremos las implicaciones educativas de estos enfoques haciendo especial énfasis en el rol de los centros de escritura como agentes dinamizadores de la renovación en las prácticas de escritura y de alfabetización académica en contextos anglosajones. Finalmente, analizaremos el estado de la cuestión en el contexto español y daremos cuenta de algunas iniciativas de investigación recientes que tienen por objetivo avanzar en nuestro conocimiento acerca de las prácticas de alfabetización académica en nuestro contexto y sensibilizar a la comunidad universitaria de los retos a los que se enfrentan profesores y estudiantes.

Palabras clave: escritura académica, escritura a través del currículo, escritura en las disciplinas, concepciones sobre la escritura

* El presente artículo hace parte del libro digital Escribir en el contexto académico [Inmaculada Ballano, Itziar Muñoz (coords.). (2014). Serie Maior, 19. Bilbao: Deusto Publicaciones, Universidad de Deusto. ISBN: 9788415759546].

1 Catedrática de la Facultad de Psicología, Ciencias de la Educación y del Deporte, Fundación Blanquerna, Universidad Ramon Llull; coordinadora del máster y el doctorado interuniversitario en Psicología de la Educación, URL; coordinadora del Seminario de Investigación Interuniversitario en Estrategias de Aprendizaje (SINTE), URL. Miembro del Comité Ejecutivo de la European Association of Research on Learning and Instruction (EARLI); miembro del Comité Ejecutivo de la European Federation of Psychology Teachers Associations (EFPTA); coordinadora del Special Interest Group on Researcher Education and Careers (SIG-REaC); editora asociada del Journal of Writing Research; miembro del consejo editorial de revistas de investigación. 


\title{
1. ANTECEDENTES Y SITUACIÓN ACTUAL DE LA ESCRITURA EN LAS UNIVERSIDADES
}

\author{
Creo más bien que se piensa a partir de lo que se escribe y no lo contrario. \\ Aragon, J. L. (1989) \\ J. L. Aragon (1989). Je n'ai jamais appris á écrire ou les Incipit. \\ En É. Béguin. Les Incipit ou les mots de la fin. Europe. 81-89
}

Los antecedentes del interés actual por la escritura y, en sentido amplio, por la alfabetización académica hay que situarlos en el ámbito anglosajón, más concretamente en el ingente número de estudios realizados en EEUU y el Reino Unido, cuyos inicios se remontan a principios del siglo XX. La influencia de estos estudios en la evolución de la investigación y enseñanza de la escritura a nivel internacional es indiscutible y su análisis insoslayable para comprender los retos actuales de la investigación (Bazerman, Little, Bethel, Chavkin, Fouquette, Garufis, 2003). Por este motivo, abordaremos en este apartado la explicación de los principales movimientos que conforman el ámbito de estudio de la escritura académica con el objetivo de clarificar su evolución y situar el panorama de la investigación actual.

En primer lugar, nos ocuparemos de describir y analizar el movimiento de escritura a través del currículo, más conocido por su denominación y siglas en inglés: Writing Across the Curriculum, WAC. En segundo lugar, abordaremos los cambios que implicó la aparición del movimiento complementario al anterior conocido como escritura en las disciplinas, en inglés Writing in the disciplines, WID. Finalmente, analizaremos las semejanzas y diferencias con uno de los movimientos más potentes en la actualidad, desarrollado originariamente en el Reino Unido, denominado Academic literacies y que, siguiendo la propuesta de algunos autores, podríamos traducir como alfabetización (o alfabetizaciones) académicas (Carlino, 2013).

El origen del movimiento Writing Across the Curriculum (en adelante WAC) es multicausal y progresivo. La mayoría de los autores coinciden en señalar tres hechos coyunturales que abonaron el terreno para que se produjera su desarrollo:

a) La progresiva masificación de la Universidad tanto en los EEUU como en el Reino Unido en la primera mitad del siglo XX. Especialmente a partir de los años 60, el acceso de los jóvenes a las Universidades aumentó de manera geométrica y este hecho, que conllevó muchas ventajas asociadas como la democratización del acceso a la educación superior, supuso también la constatación y posterior queja por parte de muchos profesores universitarios respecto a la falta de preparación de estos jóvenes que, por primera vez, no provenían exclusivamente de las elites intelectuales y educativas. Las quejas se dirigían de manera clara a sus dificultades para leer y escribir y, consiguientemente, para entender y comunicar contenidos complejos como los propios de los estudios universitarios.

b) Ante las quejas sobre las carencias de los estudiantes, muchas universidades, preferentemente en los EEUU, desarrollaron cursos de escritura que constituían un requisito previo para el acceso a determinados contenidos. Estos cursos que se desarrollaban durante el primer año (First Year Writing Courses) tuvieron inicialmente un carácter remedial y la intención genérica de preparar a los estudiantes para hacer frente a las demandas de escritura de la universidad. Muy pronto, dado que no se obtenían los resultados esperados, se complementaron con nuevas ofertas de cursos aún más básicos (de habilidades lingüísticas) dirigidos a los estudiantes que no superaban una prueba previa de acceso de las universidades (Bazerman, Little, Bethel, Chavkin, Fouquette, Garufis, 2003). 
c) En este contexto de descontento generalizado, las reformas curriculares emprendidas por el Reino Unido entre 1960 y 1970, y la investigación desarrollada por James Britton, fueron la semilla del desarrollo del movimiento WAC en EEUU, y, como veremos, en cierta medida también de otras iniciativas innovadoras del Reino Unido (Russell, 1991; Bazerman y Russell, 1994). En sus conclusiones Britton (1970) abogaba por un cambio en el enfoque de la enseñanza de la escritura argumentando que era necesario que los estudiantes escribieran textos funcionales en el seno de cada una de las materias curriculares. El trasfondo de su argumento tenía que ver con el poder epistémico del lenguaje, y más concretamente de la escritura, a la hora de aprender. Estas ideas encontraron el terreno abonado en los EEUU donde precisamente las universidades intentaban reformular los mencionados cursos de enfoque remedial.

En base a estos antecedentes, se empiezan a desarrollar los primeros programas de escritura a través del currículo (WAC) en diferentes universidades norteamericanas. De lo que se trataba inicialmente era de que los cursos de primer año existentes aumentaran su vinculación con las diferentes disciplinas y, por tanto, el cambio implicó pasar de cursos de escritura generales a cursos de escritura para historiadores, filósofos o químicos. Sin embargo, la mayoría de estos cursos estaban (y en algunos casos están todavía) adscritos a los Departamentos de Lengua y, consecuentemente, sus profesores pertenecían (y algunos todavía pertenecen) a estos departamentos, si bien en la mayoría de casos se promovió que tuvieran alguna formación adicional en la disciplina en cuestión.

Este cambio en los cursos de escritura rápidamente evidenció la necesidad de investigar y profundizar en las características de los textos relevantes en cada disciplina, así como de indagar las prácticas de escritura y los géneros asociados a las mismas, lo que dio origen a un nuevo movimiento centrado en la escritura en las disciplinas. El movimiento denominado en inglés Writing in the Disciplines (a partir de ahora WID) originalmente tuvo como objetivo la investigación de las formas textuales, los géneros y los discursos que predominan en las diferentes disciplinas. Consecuentemente, pretendía descifrar las prácticas discursivas de cada disciplina para, en un segundo momento, poder diseñar propuestas formativas disciplinares acordes con dichas prácticas de escritura. El objetivo pues, no era ya el desarrollo de cursos de escritura académica generales sino adentrarse en las particularidades de cada disciplina para ajustar los conocimientos formales y las convenciones de la lingüística a las particularidades de cada comunidad disciplinar, lo que también incluyó el análisis de los usos y funciones de la escritura de las diferentes comunidades profesionales y de investigación. Este objetivo complejo y ambicioso se concretó en dos grandes líneas de investigación todavía vigentes: a) la que se ocupa de estudiar los textos y los discursos de los profesionales representantes de una determinada disciplina, y b) la que se interroga por los textos que se escriben en las aulas universitarias cuando de lo que se trata es de aprender aquella disciplina (Russell, 1991; Camps y Castelló, 2013). El objetivo final de ambas aproximaciones es el mismo: incrustar o infusionar (Monereo, Pozo y Castelló, 2001) la formación en escritura dentro del currículo específico de cada una de las disciplinas.

Este cambio de foco del movimiento WID comportó importantes consecuencias, muchas de las cuales han configurado el ámbito de investigación de lo que actualmente se conoce como escritura académica. En primer lugar, afectó a la misma naturaleza de la noción de escritura en contextos académicos que pasó de ser considerada una habilidad lingüística más o menos sujeta al dominio de recursos retóricos o una habilidad cognitiva general, a concebirse como una actividad social y culturalmente situada que vehiculiza de manera diferencial las aportaciones disciplinares, y a la vez es un importante instrumento para aprender los contenidos y formas de pensamiento de estas disciplinas. El cambio de enfoque tuvo también 
importantes repercusiones en las propuestas educativas que, a grandes rasgos, asumieron la necesidad de focalizar la atención más allá de la fase de acceso a los estudios universitarios, entendiendo que a medida que el contenido disciplinar deviene más exigente y complejo, las prácticas de escritura se hacen también más específicas y, consecuentemente, requieren de enseñanza explícita si de lo que se trata es de que los estudiantes comprendan y utilicen de manera eficiente los contenidos y discursos disciplinares. Los iniciativas, pues, no se limitaron a cursos genéricos en el primer año, sino que aparecieron múltiples y diferentes propuestas de apoyo también —y especialmente- en estadios de formación avanzados con demandas de escritura más complejas como por ejemplo los estudios de máster y doctorado.

En segundo lugar, el movimiento WID generó una nueva serie de discusiones teóricas, educativas y de revisión de las prácticas (Russell y Cortés, 2012), útiles y necesarias, acerca de lo que en cada universidad y en cada estudio se entiende por dominio de la escritura por parte de los estudiantes. En los últimos años estas discusiones han comenzado a alinearse con las que se estaban produciendo en muchos de los países europeos, en los que los estudiantes universitarios se encuentran desde el principio inmersos en el estudio de las disciplinas sin la posibilidad de acceder a una formación más genérica en habilidades, como ocurre en el primer año del plan de estudios de muchas universidades de los EEUU (Castelló y Donahue, 2012). Sin embargo, este diálogo no está resultando fácil, no solo por las barreras idiomáticas, sino también por barreras culturales y epistemológicas menos evidentes pero igualmente importantes (Lillis y Curry 2006).

Por último, en los últimos veinte años, en los EEUU la atención se ha decantado de manera clara hacia el estudio de los géneros, entendidos, a partir de la aportación pionera de Miller (1984), como constelaciones de formas unidas por una dinámica interna, de tal manera que la dinámica 'funde' características sustantivas, estilísticas y situacionales. Desde esta perspectiva, los géneros son construcciones sociales que poseen cierta estabilidad, pero al mismo tiempo son dinámicas y cambiantes, y además devienen motivos sociales de participación (Camps y Castelló, 2013). Las investigaciones en este caso se han preocupado por analizar en qué esferas de la actividad académica el estudiante requiere comprender o producir determinados géneros y cuáles son las características estables o dinámicas de los mismos. El marco de la teoría de la actividad y las nociones de comunidades de práctica disciplinares han sido los referentes conceptuales y metodológicos de muchas de estas investigaciones en los últimos años (Russell y Cortés, 2012).

Todos estos cambios, que evidentemente se han producido de manera gradual, unidos a la globalización de la comunicación académica, han propiciado en la actualidad la emergencia de iniciativas cuyo principal interés es promover el diálogo entre comunidades internacionales geográfica y culturalmente diversas. Evidencias de algunas de estas iniciativas son las tres ediciones de la Conferencia Writing across the Borders (en 2009, 2011 y 2013) la primera que reúne científicos de los cinco continentes o algunas publicaciones recientes que han explicitado su voluntad de integrar las voces de investigadores de ambos lados del Atlántico (Bazerman, Dean, Early, Lunsford, Null, Rogers y Stansell, 2012; Castelló y Donahue, 2012; Delcambre, Lahanier-Reuter y Bart 2014).

En este diálogo, la investigación desarrollada en el Reino Unido constituye uno de los protagonistas destacados. Como hemos visto, su influencia en el desarrollo de los movimientos WAC y WID fue decisiva. Sin embargo, el movimiento iniciado en el Reino Unido ha tenido un desarrollo muy diferente $y$, a partir de 1990, se concretó en lo que algunos autores han traducido como Alfabetización Académica, o mejor dicho alfabetizaciones académicas (Academic literacies), a partir de ahora ACLITS (Russell, Lea, Parker, Street y Donahue, 2009).

El término Literacy no tiene una traducción fácil ni unívoca. Nosotros hemos optado por una de las traducciones posibles, la de alfabetización. Aunque éste es su significado literal, no es aconsejable 
equipararlo a lo que tradicionalmente se ha entendido por alfabetización en nuestro contexto, la adquisición temprana del código y de las habilidades básicas para leer y escribir. El término Literacy remite a todos los niveles educativos y tiene que ver con la formación continua en lectura y la escritura que nos convierte en personas letradas, es decir aquellas que pueden hacer uso de sus habilidades discursivas de manera activa y ajustada a las exigencias de los contextos en los que participan de tal forma que son competentes para contribuir activamente al desarrollo de su comunidad y disfrutar como miembros de pleno derecho de sus beneficios. Teniendo en cuenta este significado algunos autores han optado por traducirlo como literacidad (ver al respecto Carlino, 2013).

El concepto de alfabetización académica, tal como se usa en este texto, cobra sentido bajo la concepción que acabamos de formular y propone la enculturación académica en los códigos, discursos y convenciones disciplinares, como una forma específica de alfabetización que las instituciones de Educación Superior deberían promover para garantizar que sus estudiantes puedan ser miembros activos de $-\mathrm{y}$ por consiguiente, comunicarse de manera adecuada con- sus respectivas comunidades profesionales.

El trabajo pionero de Hounsell (1984), junto con la aportación de Britton ya mencionada, son los antecedentes inmediatos del desarrollo del movimiento ACLITS en el Reino Unido; Hounsell identificó el discurso académico como un tipo particular de escritura, con un conjunto de convenciones o códigos que le son propios, al tiempo que también subrayó su carácter tácito y los problemas de los estudiantes cuando se enfrentan por primera vez al mismo a través de las materias objeto de estudio.

Por otra parte, y de manera también similar a lo que pasó en EEUU, en los años 90 las universidades del Reino Unido, tradicionalmente elitistas y con pocos estudiantes, se masificaron al tiempo que se produjo una importante reforma del sistema que eliminó la distinción entre universidades propiamente dichas y escuelas politécnicas, que hasta entonces habían representado dos niveles diferenciados en cuanto a calidad percibida, excelencia y número de recursos. Todo ello supuso un creciente malestar y la aparición de quejas parecidas a las de EEUU respecto a la falta de preparación de los estudiantes, que propiciaron la proliferación de centros de apoyo al aprendizaje (Learning Centers), en los que se pretendía enseñar a los estudiantes las habilidades y estrategias de trabajo autónomo que reclamaba la Universidad. Uno de los problemas fundamentales que tuvieron que encarar estos centros fue el de la escritura, de tal manera que muchos de ellos se especializaron y se convirtieron en la semilla de los Writing Centers presentes actualmente en la mayoría de las Universidades del Reino Unido.

En este contexto, además de los teóricos ya señalados, el trabajo crítico de Ivanič crítico sobre el poder del lenguaje y su repercusión en la identidad del estudiante resultó clave en la orientación final de movimiento ACLITS. Sus aportaciones pusieron de manifiesto cómo la creación de identidades disciplinares son fundamentalmente discursivas y relacionales y están mediadas por las prácticas de alfabetización en las que participan (Clark e Ivanič, 1991).

Posteriormente, en 1996 Brian Street publicó un innovador capítulo sobre la alfabetización académica (Academic Literacy) desafiando, por un lado, las propias convenciones académicas (incorporando textos originales de otros en lugar de integrarlos en su discurso haciendo uso de la referencia convencional) y por otro lado, poniendo sobre la mesa cuestiones nucleares de la propia noción de alfabetización. En esta publicación (Street, 1996) retoma una cuestión que él mismo había abordado unos años antes, cuando distinguió entre dos modelos de alfabetización, el autónomo y el ideológico (Street, 1984). Mientras que un modelo autónomo de alfabetización sugiere que la alfabetización es una habilidad descontextualizada que una vez aprendida se puede transferir con facilidad de un contexto a otro, el modelo ideológico pone de relieve la preponderancia del contexto social y la naturaleza situada de las prácticas de alfabetización, 
así como las relaciones de poder y autoridad que están implícitas en cualquier situación de alfabetización y que condicionan sus resultados.

La alfabetización, así entendida, una vez adquirida no es una habilidad aplicable a cualquier contexto que requiera el dominio de la palabra escrita, todo lo contrario; dado que las prácticas de escritura y lectura son actividades profundamente sociales, solo es posible entenderlas y familiarizarse con ellas en los contextos sociales específicos en los que estas actividades tienen sentido. En estos contextos hay que tener en cuenta, además, las complejidades ideológicas que acompañan a dichas prácticas, como por ejemplo, el valor que se otorga a algunos géneros particulares por encima de otros o la preponderancia de algunas de las convenciones de los textos escritos. Como se puede deducir de lo que acabamos de comentar, la investigación realizada desde la perspectiva ACLITS se nutrió desde sus inicios de las aportaciones de la sociolingüística, la etnografía y la antropología lingüística, aportaciones que no conformaron en igual medida la evolución del ámbito de la escritura académica en los EEUU.

Han sido muchos los autores de este movimiento que en sus primeras publicaciones se dedicaron a ilustrar la multiplicidad de discursos que se producen en los contextos académicos, haciendo hincapié en la noción de "discursos" en plural, aunque hasta entonces casi siempre se había hablado de discurso, en singular. El análisis no solo se limitó a demostrar su existencia, sino también a averiguar cómo compiten estos discursos y cuáles son los mecanismos que hacen que tanto estudiantes como profesores terminen priorizando unos por encima de los otros (Ivanič, 1998; Lea, 1994; Lillis, 1997). Sin embargo, en los últimos años el foco del interés se ha centrado casi exclusivamente en los textos escritos, por lo que el término discurso —o discursos - ha sido progresivamente sustituido por el de afabetitzaciones en plural (literacies).

La investigación realizada por Lea y Street (1998) supuso un punto de inflexión del movimiento por diferentes motivos. En primer lugar, porque defendió la necesidad de examinar las expectativas y representaciones de docentes y estudiantes sin hacer juicios sobre la idoneidad de sus prácticas de escritura, alejándose así de las clasificaciones habituales entre escritores "buenos" y "no tan buenos". La hipótesis subyacente de su enfoque tenía que ver con la idea de que el sentido que ambos colectivos otorgaban a las prácticas de escritura estaba directamente relacionado con sus expectativas y representaciones, tanto de la actividad académica como de su rol en la misma. Esta fue una de las conclusiones del estudio que desarrollaron en dos universidades del Reino Unido muy diferentes, en las que analizaron qué escribían los estudiantes y enmarcaron estas prácticas en sus respectivos contextos institucionales, las relaciones de poder y las identidades. Además, los resultados pusieron de manifiesto diferencias fundamentales en el sentido que estudiantes y profesorado otorgaban a las actividades de escritura.

En segundo lugar, porque, a partir de los datos empíricos analizados, establecieron tres modelos explicativos de las concepciones sobre la escritura académica y las prácticas asociadas a los mismos. El primero, denominado técnicas o habilidades de estudio (Study skills), propugna que la buena escritura se apoya fundamentalmente en el dominio de las reglas de la gramática y la sintaxis, que pueden incluir, en situaciones académicas, los aspectos relativos a las estructuras prototípicas de los textos; por lo tanto, bajo este modelo, para asegurar la competencia de los estudiantes, basta con asegurarse de que se enseña el dominio de estas habilidades que sirven para escribir en situaciones diversas cualquier tipología de textos.

El segundo se denominó modelo de socialización académica (socialization) porque asume que la finalidad de las prácticas de escritura académica es que los estudiantes se familiaricen con los discursos y géneros específicos de las disciplinas particulares y, consecuentemente, participen de la cultura escrita de estas disciplinas. Bajo este modelo, se considera, pues, que el hecho de hacer explícitas las características y 
también los requisitos de la escritura en las diferentes disciplinas contribuirá a que los estudiantes aprendan a escribir, y a pensar, como miembros de estas disciplinas.

El tercer modelo fue específicamente denominado de alfabetización académica (Academic literacy) y, si bien no niega la pertinencia de los anteriores, añade la necesidad de tener en cuenta la naturaleza de las prácticas institucionales, las relaciones de poder que conllevan, así como el impacto en la formación de la identidad que supone su adquisición. Esto lo hace más completo y en definitiva más capaz de dar cuenta de la complejidad del sentido y el significado de la escritura en contextos académicos, aspectos que los otros dos modelos no contemplan.

La propuesta y caracterización de estos tres modelos ha sido ampliamente aceptada y utilizada tanto en la investigación como en el diseño de propuestas para la enseñanza y el aprendizaje de la escritura y representó en su momento una vía eficaz para la superación de los modelos centrados en el déficit y los cursos remediales. Su popularización ha marcado la evolución de propuestas educativas específicas de las que nos ocuparemos en el próximo apartado.

1.1. Implicaciones educativas de los movimientos WAC, WID y ACLITS: evolución y situación actual de los Centros de Escritura

La creación y desarrollo de Centros de Escritura $(\mathrm{CsE})$, más conocidos por su denominación en inglés Writing Centers (WCs) — son una de las consecuencias más palpables y consolidadas de los movimientos WAC, WID y ACLITS analizados en la sección anterior. Son predominantes en los contextos universitarios anglosajones, si bien son cada vez más numerosas las iniciativas emergentes en contextos Europeos y de América Latina.

Los orígenes de los WCs se remontan a finales del siglo XIX y principios del XX, cuando se crean los primeros "laboratorios de escritura". Aproximadamente a partir de 1920, dichos laboratorios empiezan a gozar de notable popularidad; sin embargo, en este momento se conciben más como un "método" que como un "espacio" (de hecho, en muchos casos se desarrollan en la propia aula de clase) y se centran principalmente en los aspectos gramaticales de la escritura. No es hasta la década de 1930 que en la Universidad de lowa se crea el primer Writing Lab como centro externo a la sala de clases, acercándose un poco más a la idea actual de los WCs (Waller, 2002; Murphy y Stay, 2006).

A partir de los años 1940, los laboratorios de escritura, como espacios para aprender a escribir, continúan proliferando en las universidades estadounidenses, pero en este momento lo hacen ya bajo una nueva etiqueta: "clínicas de escritura". La denominación ilustra bien el enfoque médico-remedial que los fundamenta, al que nos hemos referido en el apartado anterior. Así, la función principal de dichas "clínicas" es realizar un diagnóstico de las dificultades del alumno y la prescripción de actividades remediales que le permitan mejorar la calidad de sus textos (Waller, 2002).

Progresivamente, de manera especial a partir de 1960, el objetivo compensatorio de los laboratorios o clínicas de escritura se difumina, al tiempo que cada vez son más numerosos los alumnos que acuden a los mismos de forma voluntaria, independientemente de sus resultados académicos (Waller, 2002). En los 70, coincidiendo con las primeras investigaciones acerca de la escritura en las disciplinas, se populariza la denominación de Writing Centers, tal y como los conocemos en la actualidad (Bazerman, 2004). De forma progresiva, en la mayoría de los WCs, a la luz de los presupuestos teóricos del movimiento WAC, se empieza a abandonar la metodología mecanicista basada en técnicas y ejercicios, para basar la enseñanza de la escritura en la discusión, la revisión y el trabajo colaborativo. 
A partir de la década de 1980, de forma congruente con el desarrollo del movimiento Writing In the Disciplines (WID), el foco de los WCs se redefine y amplia, dirigiéndose al estudio y la comprensión de aspectos tales como: 1) el análisis de las formas textuales y vías de comunicación a través de las cuales las diferentes disciplinas construyen conocimiento, y 2) la caracterización de los retos a los que se enfrentan los alumnos cuando aprenden a escribir en y para cada disciplina (Bazerman, 2004).

A finales de los 80 y principios de los 90, los WCs son ya centenares en las universidades norteamericanas y las investigaciones y publicaciones científicas sobre la evolución de dichos centros se disparan, abandonando presupuestos exclusivamente cognitivos y mecanicistas para enmarcarse en un paradigma epistemológico claramente sociocultural (Russell et al., 2009). De forma congruente con esta escalada de popularidad, en el año 1983 nace la primera asociación de WCs, la National Writing Centers Association (NWCA), que, con la abertura de nuevos centros a nivel internacional, pasa a denominarse International Writing Centers Association (IWCA).

En este momento, en el contexto estadounidense, los WCs ya se habían convertido en un pilar central en la enseñanza de la escritura y adoptado las funciones que continúan ejerciendo en la actualidad: apoyar el diseño y el desarrollo de los programas curriculares; fomentar y estimular el pensamiento reflexivo de los escritores; erigirse en polos de atracción donde los escritores de la comunidad universitaria pueden encontrar lectores competentes y reflexivos para sus escritos; y ofrecer un espacio que permita hablar y conversar sobre la escritura para conseguir mejores escritores, no solamente mejores escritos (Waller, 2002).

No es de extrañar que el impacto que han tenido y siguen teniendo los WCs en Estados Unidos haya conducido a que, en esta última década, se hayan desarrollado iniciativas similares en diferentes países tanto europeos como latinoamericanos.

En América Latina, la creación de WCs es relativamente reciente y aún hoy poco extendida, aunque el crecimiento es continuo y la sensibilización de la comunidad universitaria acerca de la promoción de escritores competentes es muy elevada (Pérez-Abril y Rodríguez, 2013; Carlino, 2013). Los centros creados en la última década se han diseñado mayoritariamente a estilo y semejanza de los centros norteamericanos $y$, al menos nominalmente, persiguen objetivos similares.

En Europa, en el año 1998 nace la European Writing Centers Association (EWCA) con el objetivo de integrar los WCs de las universidades europeas en un marco común, con el objetivo de compartir recursos e información y discutir de forma colegiada sus metas y objetivos. Se convierte así en la primera asociación filial de la International Writing Centers Association (IWCA) fuera de Estados Unidos. Probablemente debido a esta afiliación, es fácil constatar la similitud de la mayoría de los principios que fundamentan los WCs europeos miembros de la EWCA, con los de los centros estadounidenses.

No obstante, en el contexto europeo, no todos los WCs han tenido una trayectoria paralela a la de Estados Unidos. En este sentido, cabe destacar el caso de los Centros que bajo denominaciones diversas han proliferado en el Reino Unido. El particular énfasis del movimiento Academic Literacies —ACLITS — en entender la naturaleza situada de la alfabetización académica, explica que la mayoría de los WCs del Reino Unido no tuviesen, ni en su origen (mucho más tardío, alrededor de 2004), ni posteriormente, el enfoque remedial y/o rehabilitador que caracterizó las primeras etapas de su desarrollo en los EEUU.

En la actualidad, muchas de las iniciativas desplegadas en el Reino Unido para fomentar la enseñanza estratégica de la escritura muestran explícitamente una orientación sociocultural y situada de la escritura, que implica el diseño de actividades y proyectos en colaboración con los profesores y especialistas de las diferentes disciplinas y que, en último término, promueve el desarrollo de la identidad de los escritores a través de la atención a las diversas alfabetizaciones que se requieren en el ámbito académico. 
La idea subyacente de este enfoque es precisamente que el éxito del aprendizaje depende del éxito de estas alfabetizaciones, es decir, de la posibilidad de que los estudiantes se comuniquen de forma adecuada y pertinente en los diferentes contextos a los que se ven expuestos y puedan usar los recursos discursivos de sus disciplinas para conseguir las finalidades epistémicas que se propongan. Un buen ejemplo, que permite visualizar una de las posibles concreciones de esta orientación, es el caso especial del proyecto Write Now, que funciona en red y lleva desarrollándose en el Reino Unido desde 2005, integrando a tres universidades: London Metropolitan university, Liverpool University y Aston University.

1.2. Principios y supuestos subyacentes a las propuestas educativas diseñadas actualmente en los WCs eficaces

En este apartado aludiremos a aquellos principios que, en base a los antecedentes teóricos revisados y los resultados de la investigación sobre el tema, nos parece que deberían constituirse en los ejes inspiradores de un Centro de Escritura eficaz.

En primer lugar nos ocuparemos de aquellos aspectos vinculados a las finalidades de un centro y al carácter o tipo de asesoramiento que se promueva desde el mismo. En segundo lugar, desglosaremos los argumentos relativos al tipo de destinatarios y al alcance de las acciones que pueden desarrollarse en relación con cada uno de los destinatarios. Por último, discutiremos los principios que subyacen a la definición de un determinado Plan de actuación y que tienen que ver con la estructura del centro y con el grado de concreción de los procedimientos que se desplieguen.

\subsubsection{Finalidades y carácter del asesoramiento}

Como hemos visto en los apartados anteriores, la historia y la evolución de los Centros de Escritura Writing Centers revela una progresión desde posiciones remediales a posiciones cada vez más preventivas y proactivas en pos de un trabajo colaborativo que mejore el aprendizaje de los estudiantes.

Desde el modelo remedial, la postura de partida implica la aceptación del hecho de que los estudiantes están poco —o mal_ preparados para afrontar las demandas de escritura que les va a plantear el contexto universitario; en definitiva, se comparte la tan cacareada expresión de que los estudiantes "no saben escribir". Dado que la finalidad de la universidad no es proveer este aprendizaje - este es otro de los supuestos fundamentales de este modelo-, se necesita de una instancia con unos profesionales específicos - preferentemente del área de lenguaje-, suficientemente preparados para proporcionar a los estudiantes la formación de la que carecen. Se trata pues de que esta instancia —el Centro de Escritura o similar-, les enseñe las habilidades básicas de escritura que deberían conocer para desarrollar su actividad como estudiantes universitarios. Además, bajo este modelo, estos profesionales, y por consiguiente también el centro, seguirán ayudando a los estudiantes a escribir sus textos y actuarán de muletilla —o de compañeros de escritura — a lo largo de los estudios universitarios, habitualmente al margen de los currículos oficiales de cada carrera y disciplina.

Desafortunadamente, los supuestos en los que se apoya este modelo han sido sistemática y reiteradamente desmentidos por la investigación en escritura en los últimos veinte años. Los numerosos trabajos, desarrollados desde diferentes perspectivas (Alamargot y Chanquoy, 2001; Albertinti, 2008; Bazerman y Russell, 2004; Bazerman, Keranen y Encinas, 2012; Camps, 1995; Camps y Castelló, 1996; Castelló et al. 2007; Castelló, 2009a; 2009b; Prior, 2006; Robinson-Pant y Street, 2012; Russell, 1991; 2007; Street, 1996; entre otros), han puesto de manifiesto que la escritura no es una habilidad general que uno adquiera de una vez por todas en momentos tempranos de su educación y que, una vez dominada, se pueda aplicar sin dificultad a cualquier 
contexto, tarea o actividad en la que se requiera. Más bien al contrario, la escritura académica tiene que ver con el dominio y ajuste de determinadas habilidades que son específicas y dependientes de los contextos en los que esta actividad tiene sentido y que, en función de las características de dichos contextos y actividades, deben desarrollarse de diferente manera para conseguir finalidades disciplinares concretas. Esto significa que hay que aprender a dominar los diferentes tipos de escritura y conocer los mecanismos discursivos y las finalidades a las que sirven en cada contexto y disciplina. Además, los mecanismos de escritura que son propios de las diferentes disciplinas están intrínsecamente vinculados a los mecanismos de construcción de conocimiento de dichas disciplinas y, dado que la adquisición y creación de conocimiento disciplinar es uno de los objetivos fundamentales de la formación universitaria, parece obvio que esta formación debería incluir también el desarrollo de los mecanismos discursivos propios de la escritura en cada una de las disciplinas.

Por otra parte, los modelos remediales de asesoramiento, también en contextos no exclusivamente vinculados a la escritura, han sido ampliamente denostados no solo por su falta de eficacia sino sobre todo por la falta de co-responsabilización que generan en los agentes educativos implicados en la resolución del problema, como por ejemplo, profesores y gestores (Castelló y Monereo, 2000; Monereo, Pozo y Castelló, 2001). Así, cuando la instancia asesora, en nuestro caso el Centro de Escritura, se erige en garante de la solución de los problemas de escritura, se produce paralelamente una desvinculación de estos problemas por parte del resto de la comunidad educativa, que considera que este no es un problema que le afecte puesto que compete solo a los profesionales especializados sobre el tema.

Sin embargo, desde esta perspectiva la frustración aparece con mucha frecuencia cuando se constata que, pese a los cursos y las propuestas de todo tipo que los profesionales especializados llevan a cabo con los estudiantes, estos no mejoran en el desempeño específico de cada una de las disciplinas. En estos casos, el descrédito del Centro de Escritura y de sus profesionales está asegurado y todo ello sin que la comunidad educativa se percate de que no se trata de que los profesionales del Centro no ejercieran bien su trabajo ni que el desarrollo de Centros de Escritura sea ineficaz en sí mismo, sino que lo que se revela inadecuado es el modelo remedial del cual se partió, modelo que deposita toda la responsabilidad en unos profesionales que deben operar al margen de las situaciones reales en las que escribir - y aprender - tiene sentido, con lo que nunca llegarán a modificar las prácticas de escritura que estudiantes y profesores despliegan en estas situaciones en las que la escritura es funcional.

El modelo de asesoramiento colaborativo, desarrollado desde presupuestos constructivistas (Monereo y Solé, 1996), plantea una perspectiva radicalmente distinta, en la que los profesionales expertos trabajan de forma coordinada y colaborativa con los profesores y demás agentes educativos implicados en la formación de los estudiantes y responsables, en último término, del diseño y evaluación de las actividades de escritura que estos deberán desplegar a lo largo de sus estudios. Este es el modelo que, con denominaciones diversas y algunos matices diferenciales, caracteriza la mayoría de las propuestas actuales de los WCs que operan en EEUU y de algunos de los más relevantes de otros contextos. En estos WCs, los profesionales que los dirigen están convencidos de que la escritura académica es una actividad situada y vinculada a la creación de conocimiento disciplinar y, por ello, diseñan sus intervenciones intentando contar con el necesario concurso de los agentes educativos implicados para que el cambio se produzca tanto en los estudiantes como en las prácticas pedagógicas y educativas de los profesores, de tal manera que se modifiquen los contextos en los que se escribe, no únicamente los textos que se producen. Esto implica trabajar de forma colaborativa, sabiendo que cualquier intervención es sistémica y debe atender a varios frentes. Exige, además, un amplio conocimiento de las concepciones y expectativas respecto a la escritura académica —y su rol en el aprendizaje- de cada uno de los agentes implicados con los que se va a colaborar (estudiantes, profesorado y gestores universitarios), así como de las respuestas y actividades habituales que cada colectivo despliega alrededor de la 
escritura colaborativa. Por último, requiere de un adecuado despliegue de mecanismos y recursos que filtren las percepciones y resultados de cada colectivo, que permita dar cuenta de los avances de forma realista y colegiada. Se trata de un modelo más complejo, pero evidentemente más eficaz, que permite cambios sostenibles y liderados de forma distribuida, de tal manera que toda la comunidad se responsabiliza de los avances y del desarrollo de la escritura académica, entendida como una herramienta epistémica que contribuye a la creación de aprendizaje.

En el ámbito universitario, evidentemente marcado por una fuerte especialización disciplinar, puede operar una necesaria división de funciones que permita acciones diferenciales con profesores y estudiantes, aunque siempre con la finalidad de que las ayudas ofrecidas redunden en un cambio de los contextos de enseñanza y aprendizaje, no solo de los productos finales - textos- que se generan en tales contextos. El objetivo a medio plazo debería ser el de crear estructuras estables de colaboración con el profesorado que permitieran actuaciones conjuntas dirigidas a ayudar a los estudiantes a gestionar mejor la escritura como una herramienta de aprendizaje, mediante la adquisición de habilidades específicas y generales, la reflexión sobre las situaciones comunicativas, el desarrollo de la propia voz y la identidad como escritores y profesionales reflexivos para que en cada disciplina puedan ser capaces de comprender y elaborar nuevo conocimiento. Estas estructuras estables de colaboración pueden permitir, a medio y a largo plazo, modificaciones sustanciales y sostenibles de las prácticas de enseñanza y aprendizaje gracias a la reflexión sobre los mecanismos implicados en el aprendizaje y comunicación disciplinar, con especial énfasis en las prácticas de escritura y lectura académicas y su uso en las situaciones habituales de enseñanza.

\subsubsection{Destinatarios de la intervención y alcance de las acciones}

A la luz de las consideraciones precedentes, parece obvio que los destinatarios de la intervención de un Centro de Escritura, o instancia similar, no deberían ser solo los estudiantes, sino que tiene sentido ampliar el foco de intervención a la comunidad universitaria en general. Esto implica obviamente acciones diferenciadas y específicas para cada uno de los colectivos que integran esta comunidad, pero también el diseño orquestado y coordinado de estas actividades específicas de tal manera que en su conjunto respondan a un marco de actuación coherente, global e integrado que incluya, por este orden, a los estudiantes, sus profesores y los gestores o autoridades universitarias.

En cuanto a los estudiantes, obviamente son el origen de las preocupaciones de un CE. Sin embargo, desde el modelo de asesoramiento colaborativo no remedial, en el que nos hemos situado, la atención a los estudiantes se entiende como contextualizada, es decir, la mejor manera de trabajar con los estudiantes es a partir del análisis del tipo de trabajos y demandas a las que deben enfrentarse en las situaciones de clase a las que asisten. Sin este conocimiento específico de las situaciones en las que escriben es difícil que la ayuda a los estudiantes sea eficaz y les permita resolver los problemas que tengan con la escritura, que son, también, problemas situados y dependientes de las situaciones en las que tienen su origen y sentido. Por lo tanto, cualquier iniciativa de trabajo, tanto a nivel de cursos, como de tutorías, talleres o asistencia personalizada, debería incluir el conocimiento detallado de las situaciones de clase en las que los estudiantes participan.

En relación con los profesores, estos son el eslabón imprescindible para que las actuaciones con los estudiantes sean no solo eficaces sino sostenibles y contribuyan a modificar en profundidad las relaciones de estos con la escritura, entendiéndola como una herramienta epistémica de valor en su crecimiento y desarrollo profesional. Sin embargo, para que este eslabón se convierta en un engranaje del cambio requerido y en un aliado de la intervención promovida desde el CE, es preciso prever y planificar varias acciones destinadas a 
conseguir no solo la adhesión del profesorado a los presupuestos del centro, sino también su complicidad y trabajo conjunto.

Entre estas acciones cobra especial importancia la explicitación, y, en su caso, cambio, de las concepciones que el propio profesorado tenga sobre la escritura académica. Es sumamente frecuente que los profesores desconozcan el rol epistémico de la escritura o que, aun conociéndolo, no se sientan implicados o capacitados para favorecer su desarrollo. La concepción más arraigada entre el profesorado es la que supone que la escritura es una habilidad independiente que los estudiantes deberían ya haber adquirido cuando llegan a sus aulas. Esta es la concepción que subyace, como hemos visto, al desarrollo del modelo remedial y, consecuentemente, este es el modelo que el profesorado espera que se despliegue desde el Centro de Escritura.

Se trata por lo tanto, en primer lugar, de sensibilizar al colectivo de profesores para que modifiquen su propia concepción de lo que es y para lo que sirve la escritura en el contexto universitario para conseguir que comprendan su responsabilidad en el diseño, gestión y evaluación de las actividades de enseñanza y aprendizaje que programan en sus clases y que tienen como soporte el uso de la escritura (y por supuesto también de la lectura). En segundo lugar, cuando ya resulta evidente el potencial epistémico para el aprendizaje disciplinar de las actividades de lectura y escritura, se trata de que este profesorado desarrolle un trabajo conjunto con los profesionales del CE que implica la reflexión sobre las actividades de lectura y escritura habituales en sus clases, los objetivos que las guían y las características de dichas actividades. Esta reflexión, en la que los profesionales del CE pueden aportar las herramientas de análisis propias de la composición escrita y la regulación de la escritura, y el profesorado aporta el conocimiento disciplinar respecto al sentido y significado de las actividades en la consecución de los objetivos, es en definitiva el objetivo último de un CE que trabaje bajo un enfoque colaborativo.

Una vez desarrollada esta reflexión y, si es necesario, modificadas o rediseñadas las actividades de lectura y escritura de una determinada asignatura, es el momento de ofrecer ayudas a los estudiantes que, en este caso, pueden ser completamente situadas (al estilo por ejemplo del ofrecimiento de profesores asistentes de escritura, una variante curricular e interesante de los tutores de escritura, que ha sido desarrollada con éxito por el centro de escritura de Dartmouth).

Los gestores y las autoridades académicas son también, en último término, destinatarios posibles y necesarios del Centro de Escritura. En este caso, se necesita contar con el concurso de este colectivo no solo para la necesaria dotación, y, en su caso, ampliación de recursos del CE previa evaluación de su eficacia, sino sobre todo para que con su apoyo y colaboración legitimen y hagan posible el funcionamiento del centro desde el modelo adoptado. Así, determinadas acciones pueden facilitar o dificultar el correcto y regular desarrollo de las actividades del centro, como por ejemplo, el establecimiento de determinados incentivos para el profesorado que asista con regularidad a los talleres programados desde el CE o que inicie proyectos de colaboración con el mismo, que impliquen modificaciones en su plan docente y su quehacer habitual. Estos incentivos pueden ser múltiples y diversos, desde modificaciones de horarios hasta publicaciones universitarias con las innovaciones docentes implementadas.

Por otra parte, el tipo de evaluaciones que se realicen del funcionamiento del centro y también de la actividad docente, debería ser congruente con el modelo adoptado, algo que requiere también de la complicidad y entendimiento con las autoridades y gestores encargados de gestionar e interpretar dichas evaluaciones. En último término, el apoyo decidido a unas determinadas propuestas de actuación del Centro de Escritura implica ineludiblemente la comprensión por parte de las autoridades académicas del sentido de dichas propuestas y las finalidades del centro, a la vez que exige el conocimiento y la comprensión completa y argumentada de los alcances y cambios que dichas actuaciones conllevan. 


\section{LA ESCRITURA ACADÉMICA EN ESPAÑA: RETOS ACTUALES}

El panorama que hemos esbozado en las secciones anteriores pone de manifiesto cómo la investigación sobre escritura y alfabetizaciones académicas en la universidad ha constituido una preocupación constante en el contexto internacional, y su desarrollo ha resultado clave para entender la evolución de la Educación Superior en dicho contexto. Sin embargo, este desarrollo no ha sido paralelo en todos los países europeos y el caso de España es un buen ejemplo de ello. Históricamente, las universidades del estado español han dedicado escasa atención a la enseñanza y el desarrollo de la lectura y escritura y, todavía en la actualidad, son numerosos los profesores universitarios que parten del supuesto compartido de que los estudiantes universitarios ya disponen de las habilidades necesarias para comprender y producir los textos y géneros que necesitarán en este contexto (Castelló, Mateos, Castells, Iñesta, Cuevas, y Solé, 2012). Sin embargo, la realidad desmiente continuamente este supuesto y las dificultades de los estudiantes españoles para comprender y producir textos académicos resultan particularmente evidentes cuando se enfrentan a la escritura de géneros complejos como artículos, proyectos de investigación o tesis de grado, master y por supuesto de doctorado (Castelló, Iñesta, Miras, Teberosky, Solé y Zanotto, 2007; Castelló, Iñesta, Liesa, Pardo y Martínez-Fernández, 2012; Castelló, Corcelles, Iñesta, Bañales y Vega, 2011; Marilyn y Larry, 2004; Pérez-Llantada, Plo, y Ferguson, 2011).

Es cierto que la sensibilización ha aumentado en los últimos años y algunos cambios coyunturales están propiciando no solo un aumento de la reflexión al respecto (Camps y Castelló, 2013) sino el desarrollo de iniciativas destinadas a la mejora de la escritura de los estudiantes (Álvarez-Angulo, 1996; Corcelles, Cano, Bañales y Vega, 2013; Oliva y Milian, 2013; Perea, 2013; Solé, Teberosky y Castelló, 2012; Tolchinsky, 2013). De forma paralela, las investigaciones relacionadas con la alfabetización académica en España se han incrementado de forma progresiva y constituyen un ámbito emergente que ha vivido una importante eclosión en los últimos años, por lo que la revisión exhaustiva de estos trabajos excede las posibilidades de este artículo. Sin embargo, aunque solo sea a título ilustrativo y de manera sucinta, no podemos dejar de mencionar algunas de las líneas de investigación que tienen una clara orientación didáctica o educativa, que hemos revisado de manera más detallada en algunos trabajos recientes (Castelló, Pardo y Fuentealba 2012; Castelló y Mateos, en prensa).

En primer lugar, destacan los estudios en los que se han desarrollado instrumentos de análisis de las concepciones y actitudes de los estudiantes sobre la escritura (Villalón y Mateos, 2009; Álvarez, Villardón y Yániz, 2010); un segundo grupo de trabajos se ha ocupado de analizar las características y exigencias de géneros específicos como la síntesis y la argumentación (Àlvarez, Villardón y Yáñiz, 2008; Mateos y Solé, 2009; Mateos, Solé, Martín y cols., 2014), haciendo además en algunos casos énfasis en sus relaciones con la adquisición de conocimiento (Solé et al., 2005; Mateos, Villalón, De Dios y Martín, 2007; Mateos, Cuevas, Martín y cols., 2011). Un tercer grupo de estudios se ha preocupado por diseñar y analizar propuestas formativas para facilitar a los estudiantes la apropiación de géneros propios del quehacer profesional (Roca de Larios y López Serrano, 2011) o de la investigación como la escritura de las tesis de grado (Castelló, Iñesta, Liesa, Pardo y Martínez-Fernández, 2012; Corcelles, Cano, Bañales y Vega, 2013) y de artículos de investigación (Castelló, Iñesta y Corcelles, 2013), relacionándolos en algunos casos con el desarrollo de comunidades de aprendizaje en las que los estudiantes puedan construir su identidad como escritores (Iñesta y Castelló, 2012; Oliva y Milian, 2013).

También desde ámbitos tan diversos como la lingüística, la enseñanza de lenguas extranjeras, la pragmática o el análisis del discurso, entre otros, se han desarrollado estudios sobre la escritura y los textos académicos que se han ocupado tanto de investigar el uso del lenguaje en áreas disciplinares y profesionales específicas, como la odontología, la informática, la química o la medicina (Cabré y de Enterria, 2007; Battaner, Atienza, 
López y Pujol, 2009; Morales y Cassany, 2008), tanto en pregrado (Alcaraz et al., 2007; Carciu, 2009; Salom et al., 2008; Reimerink, 2007) como en postgrado (Hewitt, y Felices Lago, 2010), o incluso en la comunicación social académica (Sabater et al., 2008).

Sin embargo, la mayoría de estos trabajos pertenecen a tradiciones epistemológicas, metodológicas y disciplinares diversas que, además, cuentan con sus propios canales de producción y difusión del conocimiento, lo que dificulta enormemente que se produzca el diálogo necesario para la integración de los avances y la creación de un cuerpo de conocimientos compartido. Por otra parte, son todavía escasas las iniciativas que se han ocupado de conocer el estado real de las prácticas de escritura y lectura en las universidades españolas, así como de describir las actividades que se priorizan o las percepciones sobre las mismas de profesores y estudiantes (Castelló, Mateos, Castells, Iñesta, Cuevas, y Solé, 2012; Solé y cols., 2005; Mateos, Villalón, de Dios y Martín, 2007; Perea, 2013).

En este contexto, hace ahora dos años, un grupo de investigadores preocupados por la temática, que habíamos participado en un proyecto en red a nivel europeo, ${ }^{2}$ organizamos un primer seminario de investigación sobre la enseñanza y el aprendizaje de la escritura académica ${ }^{3}$ con el objetivo de crear una red estable que nos permitiera compartir conocimiento, generar instancias de coordinación y analizar el estado de la cuestión de la investigación española en escritura académica en educación superior. Fruto de este seminario fue el desarrollo de un proyecto coordinado que pretende ser una primera aproximación a cómo estudiantes y profesores describen sus prácticas habituales de escritura y cuáles son las concepciones que sustentan estas prácticas en diferentes contextos disciplinares. En el proyecto han participado un total de 280 profesores/as y 1044 estudiantes de grado de 10 universidades españolas ${ }^{4}$ procedentes de titulaciones de diferentes campos de conocimiento (Artes y Humanidades, Ciencias Sociales y Jurídicas, Ciencias de la Salud e Ingeniería y Arquitectura). Los datos recogidos ofrecen información abundante sobre las percepciones de profesores y estudiantes acerca de aspectos tan diversos como la presencia de la escritura en los planes de estudio y en las aulas, la atención preferencial al proceso o al producto, el tipo de feedback que reciben los estudiantes, los géneros más frecuentes, el sentido que se otorga a la escritura y su valor en relación a otras competencias y las ayudas que los estudiantes consideran necesarias para su adecuado desarrollo. Si bien estamos todavía en pleno proceso de difusión de los resultados (Castelló, en prensa), de los análisis realizados destacan varias conclusiones que sintetizamos a continuación.

Las representaciones de profesores y estudiantes en relación al rol y la presencia de la escritura en sus clases difieren en función de su adscripción a una determinada área disciplinar más que en relación a la universidad de pertenencia u otras variables. Tanto los profesores como los estudiantes de Arte y Humanidades y de Ciencias Sociales consideran que escriben más, están más orientados al proceso y reciben o dan más feedback, que los representantes de otras áreas de conocimiento, como las Ingenierías o Ciencias de la Salud.

Además, la presencia de actividades de escritura se incrementa de manera significativa a medida que los estudiantes avanzan en sus estudios universitarios, independientemente de las áreas disciplinares a los que estos estudios pertenezcan. Así, tanto los estudiantes como los profesores coinciden en afirmar que se escribe más en los últimos cursos de licenciaturas o grados $\left(3^{\circ}\right.$ y $\left.4^{\circ}\right)$, y que en los estudios de doctorado o master se escribe con mayor frecuencia y cantidad que en el grado.

Sin embargo, los datos obtenidos reflejan representaciones dispares de profesores y estudiantes en relación a cómo se desarrollan estas prácticas de escritura. Mientras que los profesores afirman que ofrecen ayuda

2 European Research Network on Learning to Write Effectively (ERN-LWE). COST Action IS0703.

3 Primer seminario de investigación sobre escritura académica en los procesos de enseñanza-aprendizaje en la universidad española. Barcelona, Junio de 2012.

4 U. de Barcelona, U. Complutense, U. Autónoma de Madrid, U. de Murcia, U. Ramon Llull, U. Deusto, U. De Lérida, U. Pompeu Fabra, U. Alcalá, U. Comillas. 
a sus estudiantes acerca de cómo escribir y que les enseñan a planificar o revisar, los estudiantes no reconocen recibir estas ayudas, ni tampoco feedback sobre el proceso de escritura o sus borradores, sino solamente una calificación numérica sobre el producto final. Las representaciones también difieren en relación a las competencias de escritura. Mientras los estudiantes afirman planificar y leer todo el material antes de realizar las tareas de escritura encomendadas, sus profesores estiman que su escritura es mucho más lineal y poco vinculada con las fuentes de lectura recomendada.

Finalmente, destacan los resultados relativos a los géneros que predominan en las aulas, es decir a los textos que se escriben y las finalidades que estudiantes y profesores atribuyen a dichos textos. Los datos son contundentes y demuestran que casi la mitad de las actividades de escritura se circunscriben a la realización de ejercicios, desarrollo de explicaciones o resúmenes y toma de apuntes en clase. Se trata pues de un uso de la escritura casi exclusivamente dedicado a demostrar el conocimiento disciplinar adquirido (Gardner y Nesi, 2013), y en esto coinciden tanto estudiantes como profesores. Los datos son también coincidentes en lo que se refiere a la menor presencia de la escritura de textos vinculados con la práctica profesional, como el análisis y la redacción de informes, casos o proyectos, según los estudios. Sorprende además la escasa presencia de un tipo de escritura que tiene claramente funciones epistémicas, como la redacción de informes de investigación, revisiones especializadas de la literatura para escribir estados del arte o la lectura y redacción de artículos de investigación. Este tipo de escritura no llega al 6\% del total de lo que se escribe y se encuentra concentrada en los últimos cursos. Por otra parte, también resulta preocupante no solo la ausencia de aquellos géneros que suponen un tratamiento crítico de diferentes fuentes de información, sino la confusión por parte de ambos colectivos precisamente respecto a lo que supone argumentar críticamente. Un ejemplo ilustrativo de esta confusión es la variedad de finalidades y significados atribuidos al género ensayo por parte de profesores y estudiantes. Después que explicaran que habían escrito —o solicitado, en el caso de los profesores- un ensayo, les pedíamos que describieran cómo era este texto. Estas son algunas respuestas representativas de la confusión a la que hemos aludido:

"Se trata de un texto en el que hay que interpretar y explicar un tema apoyado en una fundamentación teórica". (Profesor 22)

"Ensayo sobre un libro. Tras la lectura del libro, exponer la visión de dicho autor sobre los retos de leer en el siglo XXI, la necesidad de ser un lector competente en diversos tipos de textos". (Estudiante 188)

Estos y otros resultados dibujan un panorama complejo que, en primera instancia, resulta necesario discernir, contrastar y comprender para, en un segundo momento, analizar las implicaciones y repercusiones en el tipo y la calidad del aprendizaje que promueve la universidad española. Un primer intento, no suficiente pero estimamos que necesario, para avanzar en esta dirección lo constituyen las diferentes publicaciones, algunas todavía en proceso de publicación fruto del proyecto comentado (Álvarez y Yáñiz, en prensa; Castelló y Mateos, en prensa; Castells, Mateos, Martin, Solé y Miras, en prensa; Corcelles, Oliva, Castelló y Milian, 2014; en prensa; López, Marín y Roca de Larios, en prensa).

No quisiéramos finalizar sin apuntar, de forma necesariamente breve, algunos de los retos a los que consideramos que se enfrenta la investigación en escritura académica en los próximos años en el contexto español. En unos casos, se trata de aspectos que, a la luz de algunos resultados como los que hemos presentado, resulta acuciante abordar si queremos avanzar en la(s) alfabetización(es) académica(s) (Robinson-Pantt y Street, 2012) de nuestros estudiantes. En otros casos, la reflexión proviene de situaciones emergentes que, a partir de los resultados de trabajos anteriores en otros contextos, nos parece que estamos en situación de anticipar. 


\subsection{El reto del conocimiento y la sensibilización}

En primer lugar, resulta todavía necesario sensibilizar a profesores y estudiantes del impacto que las prácticas de escritura y sus géneros asociados tienen —o pueden tener - en el tipo y la calidad del aprendizaje. Esta sigue siendo una asignatura pendiente en muchas universidades, y ámbitos disciplinares y/o profesionales, que es preciso promover desde diferentes instancias y mediante iniciativas diversas. El Seminario de escritura académica al que hemos aludido en este capítulo, pretende ser una contribución en la consecución de dicho objetivo.

Para ello es urgente promover trabajos de investigación que de forma contextualizada ilustren cuáles son los discursos disciplinares prioritarios en contextos profesionales y cómo, a través de la reflexión y la enseñanza de dichos discursos, es posible la aculturación y el desarrollo de la identidad profesional de nuestros estudiantes. El conocimiento relativo a las relaciones entre las prácticas de escritura, su presencia y enseñanza, y el desarrollo de la identidad profesional de los estudiantes es todavía escaso en nuestro contexto.

\subsection{El reto de la organización y difusión de propuestas de enseñanza eficaces}

El conocimiento y la sensibilización a la que aludíamos en el apartado anterior cobra todo su sentido cuando revierte en las prácticas de enseñanza y aprendizaje. Este es otro de los retos de la investigación en escritura de la universidad española, el del análisis, y, en su caso, modificación, de aquellas actividades de escritura que habitualmente llevan a cabo nuestros estudiantes. Cuándo y por qué se escribe un informe, un proyecto, una reseña o un artículo, cómo se incardinan estas actividades en el desarrollo de una determinada materia, cómo se evalúa o garantiza la progresión en su aprendizaje, son algunos de los aspectos que reclaman atención a la luz de algunos de los resultados de la investigación realizada en nuestro contexto.

Este primer paso relativo al análisis de las prácticas actuales tiene por objetivo final el diseño, organización y difusión de propuestas de enseñanza eficaces que garanticen un uso de la escritura como herramienta de aprendizaje. En los últimos años, como hemos visto en los apartados anteriores, se han desarrollado algunas investigaciones que avalan propuestas de este tipo tanto en grado como en postgrado. Por otra parte, el requisito de que los estudiantes dominen la escritura de géneros extensos y complejos como los trabajos finales de grado o de máster, ha generado la necesidad de reflexionar sobre los procesos de enseñanza y guía del proceso de escritura de estos textos, así como de la propia naturaleza de los mismos. Este es uno de los temas emergentes en la universidad española, que preocupa y ocupa a investigadores y profesorado, debido tanto a las dificultades que experimentan los estudiantes como a la falta de tradición en el acompañamiento de este tipo de trabajos escritos.

\subsection{El reto de la evaluación}

Por último, en los próximos años será necesario abordar con energía el análisis de los procedimientos de evaluación de los trabajos escritos que solicitamos a nuestros estudiantes. Los datos de que disponemos indican que el feedback que el profesorado proporciona no se produce durante el proceso de composición, ni resulta demasiado informativo dado que acostumbra a reducirse a una nota global sobre el texto final. Por otra parte, la creciente presencia de procesos de evaluación formativa tanto en los grados como en los masters también alude a la necesidad de consensuar y sistematizar propuestas sostenibles, efectivas y válidas de evaluación tanto de los productos finales - textos - como de los procesos seguidos para su desarrollo. Dado que uno de los argumentos sostenidos a lo largo de esta contribución es precisamente que los géneros movilizan formas 
de acción y pensamiento, la evaluación de los mismos no es un tema menor y tiene, como toda evaluación, efectos retroactivos sobre la actuación y el pensamiento disciplinar de nuestros estudiantes, lo que nos sitúa de nuevo ante el reto de la sensibilización acerca del impacto que las prácticas de escritura y los géneros asociados a las mismas tienen —o pueden tener- en el tipo y la calidad del aprendizaje y, de manera circular, nos recuerda la cita con la iniciábamos este texto: Se piensa a partir de lo que se escribe y no lo contrario.

\section{REFERENCIAS}

Alamargot, D. \& Chanquoy, L. (2001). Through the Models of Writing. Dordrecht: Kluwer Academic Publishers.

Albertinti, J. (2008). Teaching of writing and diversity: Access, identity, and achievement. En A. C. Bazerman (Ed.). Handbook of Research on Writing. History, Society, School, Individual, Text. New York \& London: Lawrence Erlbaum.

Alcaraz, E., Mateo, J., y Yus, F. (2007). Las lenguas profesionales y académicas (1 ${ }^{\text {a }}$ ed.). Barcelona: Ariel.

Álvarez-Angulo, T. (1996). El texto expositivo-explicativo: su superestructura y características textuales. Didáctica de la lengua y la literatura, 8, 29-44.

Álvarez, M. y Yáñiz, C. (en prensa). Las prácticas escritas en la universidad española. Cultura \& Educación (Submitted)

Álvarez, M., Villardón, L. y Yániz, C. (2008). Tareas de escritura en el ámbito universitario e implicaciones para el aprendizaje. Textos de didáctica de la lengua y la literatura, 49, 77-88.

Álvarez, M., Villardón, M.L. y Yániz, C. (2010). Influencia de factores sociocognitivos en la calidad de la escritura en los estudiantes universitarios. Educatio Siglo XXI, 28(2), 181-204.

Battaner, P., Atienza, E., López, C., y Pujol, M. (2009). Características lingüísticas y discursivas del texto académico. Textos de didáctica de la Lengua y la Literatura, 50, 47-67.

Bazerman, C. (2004). Intertextuality: How texts rely on other text. En A. C. Bazerman \& P. Prior (Eds.). What Writing Does and How It Does It. An Introduction to Analyzing Texts and Textual Practices (pp. 83-96). Mahwah, New Jersey: Lawrence Erlbaum Associates.

Bazerman, C., Keranen, N. \& Encinas, F. (2012). Facilitated immersion at a distance in second language scientific writing. En A. M. Castelló \& C. Donahue (Eds.). University Writing: Selves and Texts in Academic Societies, Volume Series in Writing (235-248). London: Emerald Group.

Bazerman, C., \& Russell, D. R. (Eds.). (1994). Landmark Essays on Writing Across the Curriculum (Vol. 6). Mahwah, New Jersey: Lawrence Erlbaum Associates.

Bazerman, C., Dean, C., Early, J., Lunsford, K. Null, S., Rogers, P. \& Stansell, A. (Eds.). (2012). International Advances in Writing Research: Cultures, Places, Measures. Perspectives on Writing. Fort Collins, Colorado: The WAC Clearinghouse/ Parlor Press. Recuperado de http://wac.colostate.edu/books/wrab2011

Bazerman, C. \& Russell, D. (Eds.). (2004). Writing Selves, Writing Societies: Research from Activity Perspectives. Fort Collins, CO: The WAC Clearinghouse and Mind, Culture, and Activity.

Bazerman, C., Little, J., Bethel, I., Chavkin, T., Fouquette, D. \& Garufis, J. (2003). Reference Guide to Writing Across the Curriculum. West Lafayette, Indiana: Parlor Press.

Britton, J. (1970). Language and Learning. London: Allen Lane.

Cabré, M. T. y de Enterría, J. G. (2007). La enseñanza de los lenguajes de especialidad: la simulación global. IBÉRICA, 13, 181-194.

Camps, A. y Castelló, M. (Coords.). (2013). Monográfico: Escritura académica. Academic Writing. REDU. Revista de Docencia Universitaria, 11(1). Recuperado de http://www.red-u.net/redu/index.php/REDU/issue/view/70

Camps, A. (1995). Aprender a escribir textos argumentativos: características dialógicas de la argumentación escrita. Comunicación, Lenguaje y Educación, 25, 51ه63. 
Camps, A. y Castelló, M. (1996). Las estrategias de enseñanza-aprendizaje en la escritura. En A. C. Monereo y I. Solé (Coords.). El asesoramiento psicopedagógico: una perspectiva profesional y constructivista (321-342). Madrid: Alianza

Carciu, O. M. (2009). An intercultural study of first-person plural references in biomedical writing. Ibérica: Revista De La Asociación Europea De Lenguas Para Fines Específicos (AELFE), 18, 71-92.

Carlino, P. (2013). Alfabetización académica diez años después. Revista mexicana de investigación educativa, 18(57), 355-381.

Castelló, M. (2009a). Escribir trabajos de investigación con alumnos de grado. Didáctica de la Lengua y de la Literatura, 50, 21-29.

Castelló, M. (2009b). Aprender a escribir textos académicos ¿Copistas, escribas, compiladores o escritores? En J. I. Pozo y M. del Puy Pérez (Eds.). Psicología del aprendizaje universitario: la formación en competencias (120-133). Madrid: Morata.

Castelló, M. (Coord.); Iñesta, A., Miras, M., Solé, I., Teberosky, A. y Zanotto, M. (2007). Escribir y comunicarse en contextos científicos y académicos. Conocimientos y estrategias. Barcelona: Graó.

Castelló, M. (en prensa). Escribir en la Universidad. Análisis de las concepciones, ideas y creencias de estudiantes y profesores [Writing at University. Analyzing Faculty's and Students' Conceptions, Ideas and Beliefs]. Cultura \& Educación.

Castelló, M. y Camps, A. (en prensa). Escribir en la Universidad. La perspectiva de los protagonistas: estudiantes y profesores. Cultura \& Educación.

Castelló, M. \& Donahue, C. (Eds.). (2012). University Writing: Selves and Texts in Academic Societies. Volume Series in Writing. Bingley, UK: Emerald Group Publishing Limited.

Castelló, M. y Mateos, M. (en prensa). Las representaciones de profesores y estudiantes sobre la escritura académica en las universidades españolas. Cultura \& Educación.

Castelló, M. y Monereo, C. (2000). Las concepciones de los profesores sobre la enseñanza de estrategias de aprendizaje. Ensayos y Experiencias, 33, 78-92.

Castelló, M. Iñesta, A. \& Corcelles, M. (2013). Ph.D. students' transitions between academic and scientific writing identity: Learning to write a research article. Research in the Teaching of English, 47, 442-478.

Castelló, M., Corcelles, M., Iñesta, A., Bañales, G. y Vega, N. (2011). La voz del autor en la escritura académica: una propuesta para su análisis. Signos, 44(76), 105-117.

Castelló, M., Iñesta, A., Pardo, M., Liesa, E. y Martínez-Fernández R. (2012). Tutoring the end-of-studies dissertation: Helping psychology students find their academic voice. Higher Education, 63(1), 97-115.

Castelló, M., Mateos, M., Castells, N., Iñesta, A., Cuevas, I. \& Sole, I. (2012). Academic writing practices in Spanish universities. Electronic Journal of Research in Educational Psychology, 10(2), 569-590.

Castelló, M., Pardo, M. y Fuentealba, O. (2012). Enseñar a escribir textos científico-académicos en el contexto español de investigación sobre escritura académica. Intercompreensão, 16, 45-66.

Castells, N., Mateos, M., Martín, E., Solé, I. y Miras, M. (en prensa). Perfiles de competencias y concepciones acerca de la escritura académica de los estudiantes universitarios. Cultura \& Educación.

Clark, R., \& Ivanič, R. (1991). Consciousness-raising about the writing process. En P. Garrett \& C. James (Eds.). Language Awareness in the Classroom. London: Longman.

Corcelles, M., Oliva, A., Castelló, M. \& Milian, M. (en prensa). Escribir en la Universidad: ¿nos entendemos? Cultura \& Educación.

Corcelles, M., Cano, M., Bañales, G. y Vega, N.A. (2013.). Enseñar a escribir textos científico- académicos mediante la revisión colaborativa: El trabajo final de grado en Psicología. Revista de Docencia Universitaria. REDU, Número monográfico dedicado a Academic Writing. 11(1), 79-104. Recuperado de http://www.red-u.net/

Delcambre, I., Lahanier-Reuter, D. \& Bart, D. (2014). International Studies on Writing at University: Comparisons and Evolutions. International Exchanges on the Study of Writing. Recuperado de http://wac.colostate.edu/books/international.cfm 
Hewitt, E., \& Felices Lago, A. (2010). Academic style and format of doctoral theses: The case of the disappearing discussion chapter. Ibérica: Revista de la Asociación Europea de Lenguas para Fines Específicos (AELFE), 19, 19-140.

Hounsell, D.J. (1984). Learning and essay writing. En F. Marton, D. Hounsell \& N. Entwistle (Eds.). The Experience of Learning (103-123). Edinburgh: Scottish Academic Press.

Iñesta, A. \& Castelló, M. (2012). Towards an integrative unit of analysis: Regulation episodes in expert research article writing (Chapter, 24). En C. Bazerman, C. Dean, J. Early, K. Lunsford, S. Null, P. Rogers \& A. Stansell (Eds.). International Advances in Writing Research: Cultures, Places, Measures (421-448). Recuperado de http://wac.colostate.edu/books/ wrab2011/chapter24.pdf

Ivanič, R. (1998). Writing and Identity: The Discoursal Construction of Identity in Academic Writing. Amsterdam: John Benjamins.

Lea, M (1994). I thought I could write till I came here: Student writing in higher education. En G. Gibbs (Ed.). Improving Student Learning: Theory and Practice. Oxford: OSCD.

Lea, M., \& Street, B.V. (1998). Student writing in higher education: An academic literacies approach. Studies in Higher Education, 23(2), 157-172.

Lillis, T. (1997). New voices in academia? The regulative nature of academic writing conventions. Language and Education 11(2), 182-199.

Lillis, T. \& Curry, M. J. (2006). Professional academic writing by multilingual scholars: Interactions with literacy brokers in the production of English-medium Texts. Written Communication, 23, 3-35.

López, S. Marín, J. y Roca de Larios, J. (en prensa). Qué es y qué supone escribir desde la perspectiva de profesores y estudiantes. Cultura \& Educación.

Marilyn K. F. \& Larry D. Y. (2004). Learning to write like a scientist: Coauthoring as an enculturation task. Journal of Research in Science Teaching, 41(6), 637-668.

Mateos, M. \& Solé, I. (2009). Synthesizing information from various texts: a study of procedures and products at different educational levels. European Journal of Psychology of Education, 24(4), 435-451.

Mateos, M., Cuevas, I., Martín, E., Martín, A., Echeita, G. \& Luna, M. (2011) The role of epistemological, reading and writing beliefs on reading to write an argumentation. Journal of Research in Reading, 34, 281-297.

Mateos, M., Solé, I., Martín, E., Miras, M., Cuevas, I. \& Castells, N. (2014). Reading and writing to learn in academic contexts: Writing from multiple sources. En P. Klein, P. Boscolo, L. Kirkpatrick, \& C. Gelati (Eds.). Writing as a Learning Activity. Studies in Writing Series. United Kingdom: Brill.

Mateos, M., Villalón, R., de Dios, M.J. \& Martín, E (2007). Reading-and-writing tasks on different university degree courses: What do the students say they do? Studies in Higher Education, 32(4), 489-510.

Monereo, C; Pozo, J. I. y Castelló, M. (2001). La enseñanza de estrategias de aprendizaje en el contexto escolar. A Coll, C.; Palacios, J. y Marchesi, A. (Coord.). Psicología de la educación escolar (235-258). Madrid: Alianza Editorial.

Monereo, C. y Solé, I. (1996). Asesoramiento psicopedagógico: una perspectiva profesional y constructivista. Madrid: Alianza editorial.

Morales, O., \& Cassany, D. (2008). Interpretaciones de la atenuación en artículos de revisión de Odontología. Signos, 41(67), 299-328.

Murphy, C., \& Stay, B. (Eds.). (2006). The Writing Center Director's Resource Book. Routledge.

Oliva, A. \& Milian, M. (2013). First year students' construction of an academic identity in English as a foreign language. Revista de Docencia Universitaria. REDU. Número monográfico dedicado a Academic Writing, 11(1), 59-77. Recuperado de http://www.red-u.net/

Perea, F.J. (Coord.). (2013). Comunicar en la universidad. Descripción y metodología de los géneros académicos. Ediciones de la Universidad de Córdoba: Córdoba. 
Pérez-Abril, M. y Rodríguez, A. (2013). ¿Para qué se lee y se escribe en la universidad colombiana? Caracterización de prácticas de lectura y escritura en 17 universidades. Revista de Docencia Universitaria. REDU. Número monográfico dedicado a Academic Writing. 11(1), 137-160. Recuperado de http://www.red-u.net/

Pérez-Llantada, C., Plo, R., \& Ferguson, G. R. (2011). You don't say what you know, only what you can: The perceptions and practices of senior Spanish academics regarding research dissemination in English. English for Specific Purposes, 30(1), 18-30.

Prior, P. (2006). A sociocultural theory of writing. En C. A. McArthur, S. Graham, \& J. Fitzgerald (Eds.). Handbook of Writing Research (54-65). New York: The Guilford Press.

Reimerink, A. (2007). The use of verbs in research articles: A corpus analysis. Terminology, 13(2), 177-200.

Robinson-Pant, A. \& Street, B. (2012). Students' and tutors' understanding of 'new' academic literacy practices. En A. M. Castelló \& C. Donahue (Eds.). University Writing: Selves and Texts in Academic Societies (71-92). Volume Series in Writing. London: Emerald Group Publishing Limited.

Roca de Larios, J. y López Serrano, S. (2011). Consideraciones prácticas sobre la elaboración del portafolios del estudiante: su aplicación al aprendizaje de la pronunciación del inglés. Didáctica. Lengua y Literatura, 23, 349-367.

Russell, D. R. (1991). Writing in the Academic Disciplines, 1870-1990: A Curricular History. Carbondale, IL: Southern Illinois University Press.

Russell, D. R. (2007). Rethinking the articulation between business and technical communication and writing in the disciplines useful avenues for teaching and research. Journal of Business and Technical Communication, 21(3), 248-277.

Russell, D. R. \& Cortés, V. (2012). Academic and scientific texts: the same or different communities? En A. M. Castelló \& C. Donahue (Eds.). University Writing: Selves and Texts in Academic Societies (3-18). Volume Series in Writing. London: Emerald Group Publishing Limited. (Forthcoming).

Russell, D. R.; Lea, M.; Parker, J.; Street, B. \& Donahue, T. (2009). Exploring notions of genre in 'academic literacies' and 'writing across the curriculum': approaches across countries and contexts. En C. Bazerman, A. Bonini, \& D. Figueiredo, (Eds.). Genre in a Changing World. Perspectives on Writing (459-491). Colorado: WAC Clearinghouse/Parlor Press.

Sabater, C. P., Turney, E., \& Fleta, M. B. M. (2008). Orality and literacy, formality and informality in email communication. Ibérica: Revista de la Asociación Europea de Lenguas para Fines Específicos (AELFE), 15, 71-88.

Salom, L., Soler, C., \& Carbonell, M. (2008). The move-step structure of the introductory sections of Spanish PHD theses. Revista Española de Lingüística Aplicada, 21, 85-106.

Solé, I., Mateos, M., Miras, M., Martín, E., Castells, N., Cuevas, I. y Graciá, M. (2005). Lectura, escritura y adquisición de conocimientos en Educación Secundaria y Educación Universitaria. Infancia y Aprendizaje, 28, 329-347

Solé, I., Teberosky, A. \& Castelló, M. (2012) Academic Communication Strategies in Postgraduate Studies. En C. Thaiss, G. Bräuer, P. Carlino, L. Ganobcsik-Williams \& A. Sinha (Eds.). Writing Programs Worldwide: Profiles of Academic Writing in Many Places. Fort Collins, Colorado: The WAC Clearinghouse/Parlor Press. Recuperado de http://wac.colostate.edu/ books/wrab2011

Street, B. (1984). Literacy in Theory and Practice. Cambridge: Cambridge University Press.

Street, B. (1996). Academic Literacies. En D. Baker, J. Clay \& C. Fox (Eds.). Alternative Ways of Knowing: Literacies, Numeracies, Sciences (101-134). New York: Falmer Press.

Tolchinsky, L. (Coord.) (2013). La escritura académica en las disciplinas. Barcelona: Octaedro/ICE.

Villalón, R. y Mateos, M. (2009). Concepciones sobre la escritura académica: Cómo conciben los estudiantes la escritura académica. Infancia y Aprendizaje, 32(2), 219-232.

Waller, S. (2002). A Brief History of University Writing Centers: Variety and Diversity. New Foundations. Recuperado de http://www.newfoundations.com/History/WritingCtr.html

\section{(C) $(1) \Theta$}

